\title{
Effect of biological treatment and ultraviolet (UV)-C radiation disinfection process on wastewater bacterial community as assessed by denaturing gradient gel electrophoresis (DGGE) fingerprints
}

\author{
Yousra Turki ${ }^{1 \star}$, Ines Mehri ${ }^{1}$, Hanene Cherif ${ }^{2}$, Abdennaceur Hassen $^{1}$ and Hadda Ouzari ${ }^{2}$ \\ ${ }^{1}$ Water Research and Technology Centre (C.E.R.T.E), Borj Cédria Technology Park, P.O. BOX 273, \\ Soliman 8020, Tunisia. \\ ${ }^{2}$ Laboratoire Microorganisme and Biomolécules Actives, Département de Biologie, Faculté des Sciences de Tunis, \\ Campus Universitaire, 2092, Tunis, Tunisie.
}

Accepted 20 September, 2013

\begin{abstract}
The rotating biological contactor (RBC) process was frequently used for the biological wastewater treatment in order to remove pollutants and to improve the water quality before discharge to the environment. The presence of bacteria species in the secondary treated wastewater indicates the necessity of a tertiary treatment process [ultraviolet (UV)-C radiation disinfection] to reduce the number of living organisms in the water. Denaturing gradient gel electrophoresis (DGGE) method using 16S rDNA was commonly used for a direct comparison of structural diversity among different microbial communities. In the present study, community in treated and untreated wastewater from RBC treatment plant was investigated using DGGE coupled with sequence analysis of 16S rRNA gene fragments from bands of interest. The analysis of the DGGE profiles and the sequence of the dominant DGGE bands showed a variability of the bacterial community with season. DGGE patterns of samples collected in summer were more complex than those collected in winter. In addition, the investigation of the effect of increasing $\mathrm{UV}_{\mathbf{2 5 3 . 7}}$ germicidal doses on the bacterial community, in secondary treated wastewater effluent, revealed variability in bacterial tolerance to $U V_{253.7}$ radiation. This variability is inter-specific and is dependent on the UV-C dose used and the bacterial specie irradiated. Consequently, this study demonstrated that DGGE method coupled with sequencing provides precise information on RBC and UV-C wastewater treatment process.
\end{abstract}

Key words: Wastewater, biological treatment, bacterial communities, polymerase chain reaction - denaturing gradient gel electrophoresis (PCR-DGGE), 16S rDNA, ultraviolet (UV)-C radiation disinfection.

\section{INTRODUCTION}

Wastewater and drinking water are treated to eliminate pathogenic microorganisms and to prevent waterborne transmission. However, previous study indicated that conventional wastewater treatment does not guarantee their complete elimination (Howard et al., 2004). When discharged to environment, untreated or insufficiently treated wastewaters cause several problems, such as eutrophication, oxygen consumption and toxicity (Ding et al., 2011).

The rotating biological contactor (RBC) treatment 


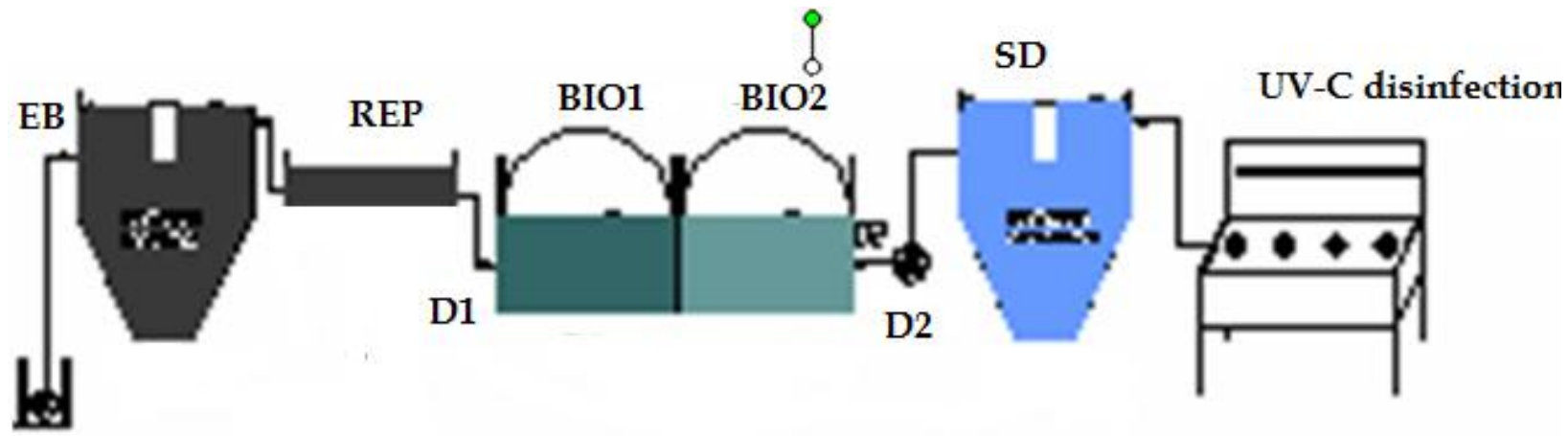

Figure 1. Experimental RBC treatment system. EB: entrance of the primary settlement tank, REP: outlet part of the primary settlement tank, D1: entrance of the first RBC tank, D2: outlet part of the second RBC tank, SD: outlet part of the secondary settling tank, $\mathrm{BIO} 1$ and $\mathrm{BIO}$ : biofilm samples taken from the first and the second disc, respectively.

process is considered one of the most frequently used methods to treat municipal wastewater. This process involves allowing the wastewater to come in contact with a biological medium in order to remove pollutants in the wastewater before discharge of the treated wastewater to the environment. In order to improve the microbiological wastewater quality, the ultraviolet (UV)-C radiation is suggested as one of the successful disinfection practices for water treatment. UV-C disinfection of water employs low-pressure mercury lamps. The lamps generate shortwave UV radiation at $253.7 \mathrm{~nm}$ which is lethal to microorganisms including bacteria, protozoa, viruses, yeasts, fungi, nematode eggs and algae. The mechanism of micro-organisms destruction is currently believed to be that in which UV causes molecular rearrangements in DNA and RNA, which is turn blocks replication (Eccleston, 1998). In fact, biological inactivation by UVC light arises from the fact that DNA molecules absorb UV photons between 200 and $300 \mathrm{~nm}$, with peak absorption at almost $260 \mathrm{~nm}$. This absorption creates damage in the DNA by altering nucleotide base pairing; thereby creating new linkages between adjacent nucleotides on the same DNA strand (Ben said et al., 2011).

Community-level studies are relying more and more on culture-independent methods based on the direct analysis of DNA or RNA without any culturing step (Jany and Barbier, 2008). Coupled to sequencing, these methods make it possible to investigate complex microbial communities. Denaturing gradient gel electrophoresis (DGGE) technique based on 16S rDNA gene was commonly used for a direct comparison of structural diversity among different microbial communities. Comparative analyses with nucleotide databases and phylogenetic reconstruction of the amplified 16S rRNA genes from DNA fragments excised from DGGE gels allowed the identification of organisms affected by the population changes (Ding et al., 2011).

In the present study, we aimed to assess bacterial community structure in wastewater and biofilm during the rotating biological contactor treatment process (second- dary water treatment process) by using DGGE and $16 \mathrm{~S}$ rRNA techniques; and to determine the influence of ultraviolet disinfection system (UV-C dose) as tertiary water treatment method on bacterial populations.

\section{MATERIALS AND METHODS}

Experimental rotating biological contactor (RBC) treatment system

Wastewater and biofilm samples were collected from Wastewater Treatment Plant located in El Menzah 1, Tunis, Tunisia (http://www.certe.rnrt.tn/station.htm), which receives wastewater of domestic origin. The Rotating Biological Contactor Process is a biological treatment process that involves the biological degradation of the wastewater pollutants and the organic material.

\section{Wastewater and biofilm sampling}

Wastewater and biofilm samples were collected from seven different points of the system. Wastewater samples for DNA extraction were collected in sterile bottles and frozen at $-20^{\circ} \mathrm{C}$ for immediate processing. Five individual well-mixed wastewater samples were taken from entrance of the primary settlement tank (EB), outlet part of the primary settlement tank (REP), entrance of the first RBC tank (D1), outlet part of the second RBC tank (D2), and outlet part of the secondary settling tank (SD). Two individual biofilm samples were taken on the first disc (BIO1) and the second disc (BIO2) (Figure 1). Samples were carried out during summer season (July: water temperature was between 30 to $32^{\circ} \mathrm{C}$ ) and winter (November: water temperature was between 20 to $23^{\circ} \mathrm{C}$ ), in order to investigate the community structure changes.

\section{Ultraviolet (UV)-C radiation photoreactor system}

The batch laboratory UV-device was built in cooperation with Guy Daric S. A. (Aubervilliers, France). This prototype contained a sliding rack, with an irradiation board that held six Petri dishes (90 $\mathrm{mm}$ diameter). A germicidal low-pressure mercury vapor discharge lamp (length $=900 \mathrm{~mm}$, diameter $=13 \mathrm{~mm}$, power of UV emission at $253.7 \mathrm{~nm}=55 \mathrm{~W}$ ) with reflector could be adjusted to different heights above the irradiation board.. Incident intensity UV rays at $253.7 \mathrm{~nm}$ were measured using a selective detector for UV joined 
to a radiometer (Vilbert-Lourmat, Norme La Vallée, France). Doses were calculated as the product of radiation intensity and time following the formula recognized by Hassen et al. (2000). In order to evaluate community resistance to UV-C light in waste water, one sample collected from secondary treatment, was irradiated (several exposure time: $30,60,90,120$ and $180 \mathrm{~s}$ ) using the photoreactor described above.

\section{Total DNA extraction}

The bacterial biomass was collected by centrifugation of $30 \mathrm{ml}$ well mixed wastewater samples at $14000 \mathrm{rpm}$ for $15 \mathrm{~min}$. Each pellet was mixed with $1 \mathrm{ml}$ of lysis buffer (50 mM Tris- $\mathrm{HCl}, 50 \mathrm{mM}$ EDTA, SDS $3 \%, \mathrm{pH} 8.0$ ). The genomic DNA of each sample was extracted with a modified method described previously (Guillaume et al., 2000).

Total DNA was extracted from biofilm samples using the fast DNA Spin Kit for Soil (MOBIO) according to the manufacture procedure. The quality of extracted DNA was analyzed by electrophoresis in $0.5 x$ TBE (Tris-Borate-EDTA buffer) on $0.8 \%$ agarose gel.

\section{PCR amplification of 16S rDNA}

Polymerase chain reaction (PCR) amplification targeting bacterial $16 S$ rDNA gene fragments was performed using the universal primers specific to the bacteria domain: 907R (5'CCGTCAATTCCTTTGATGTTT-3') and 357F (5'TACGGGAGGCAGCAG-3'). The amplified sequence is corresponding to the V3-V5 hypervariable region of $16 \mathrm{~S}$ rRNA molecule of Escherichia coli. A 40-bp GC-rich sequence (5' CGCCCGCCGCGCCCCGCGCCCGGCCCGCCGCCCCCGCCCC CC- 3') attached to the 5' end of the forward primer in order to increase separation of DNA bands in DGGE analysis Muyzer and DeWall (1993). PCR experiments were performed as previously described Sass et al. (2001).

\section{Analysis of PCR products by denaturing gradient gel electrophoresis (DGGE)}

DGGE analysis was performed by using the INGENY phorU-2 system. DGGE were applied onto $7.5 \%$ (W/V) polyacrylamide gels in $1 \mathrm{X}$ TAE $(20 \mathrm{mM}$ Tris, $10 \mathrm{mM}$ acetate, $0.5 \mathrm{mM}$ EDTA, PH 7,4) with a denaturing gradient ranging from 40 to $60 \%$. Electrophoresis was performed for $18 \mathrm{~h}$ at $99 \mathrm{~V}$. The temperature was set at a constant of $60^{\circ} \mathrm{C}$. After electrophoresis, polyacrylamide gels were stained with ethidium bromide for $20 \mathrm{~min}$. Each stained gel was immediately photographed on a UV trans-illumination table with a video camera module.

Recuperation of bands from denaturing gradient gel electrophoresis (DGGE) gels and sequence analysis

Dominant DGGE bands were selected and excised from the gel for nucleotide sequence determination. DNA of ach fragment selected was eluted by incubation in $80 \mu \mathrm{l}$ of sterilized water at $37^{\circ} \mathrm{C}$ for $7 \mathrm{~h}$ with weak shake at $450 \mathrm{rd} \mathrm{min}^{-1}$. Fifteen $\mu \mathrm{l}$ of the supernatant solution was used as a template to reamplify the band of interest using the forward primer $357 \mathrm{~F}$ without the $\mathrm{GC}$ clamp and the reverse primer 907R. A slightly different PCR protocol was used (Marzorati et al., 2006). Thereafter, the amplicons were visualized on ethidium bromide stained agarose gel, purified using the Wizard® SV Gel and PCR Clean-UP System (Promega, Madison, USA) and then sequenced by Center of BioTechnologies Sfax
(CBS), Tunisia. The sequences obtained from exciced bands in the DGGE gel were compared with the $16 \mathrm{~S}$ rDNA sequences in the GenBank database by using the basic local alignment search tool (BLAST, National Centre for Biotechnology Information, US National Library of Medicine) to recover similar sequences and phylogenetically related species. Water bacterial diversity was expressed by the Shannon diversity index $(H)$, wich was calculated as described by Steele et al. (2005).

\section{RESULTS AND DISCUSSION}

\section{Denaturing gradient gel electrophoresis (DGGE) patterns analysis of wastewater and biofilm in summer and winter}

Analysis of wastewater and biofilm DGGE patterns was based on the variation in bands number and intensity. Visual comparison of the DGGE profiles of wastewater samples and biofilm collected from different points of the RBC revealed important variations in microbial community structure between summer and winter. These changes were noted in band position, intensity, and number of bands present in bacterial DGGE patterns. In addition, analysis of DGGE patterns showed that profiles of samples collected in summer (Figure 2A) were more complex than those collected in winter (Figure 2B). As shown in Table 1, the mean value of DGGE band numbers and the Shannon diversity index decreased from $\sim 7$ to $\sim 3$ and from 1.86 to 1.09 , respectively suggesting that bacterial community diversity was affected. The probable reason of community changes may be the perturbation caused by the climatic changes, particularly the temperature (Gilbride et al., 2006; Moura et al., 2009; Ding et al., 2011).

In summer, the results showed that considerable differences in bacterial DGGE data were observed between different points of the process. More bands were detected in samples from the out part of the bioreactor (SD) than those from the entrance of the bioreactor (EB, REP, and D1) and from biofilms (BIO1, BIO2). These results suggested that bacterial populations were accumulated in the secondary settling tank.

In winter, a decrease in bands number and intensity were observed in DGGE community patterns. Summer bacterial community was disappeared and yielded places to novel bacterial populations (B16 and B18). This result suggested that the bacterial community diversity were affected by the period of the year.

\section{Sequence analysis of the dominant bacteria in the secondary treated wastewater}

In order to determine dominant bacterial species in the secondary treated wastewater (the outlet part of the secondary settling tank), a number of 8 bands showing the highest intensity from gels were excised, re-amplified, purified, sequenced and subjected to BLAST GeneBank 
(A)

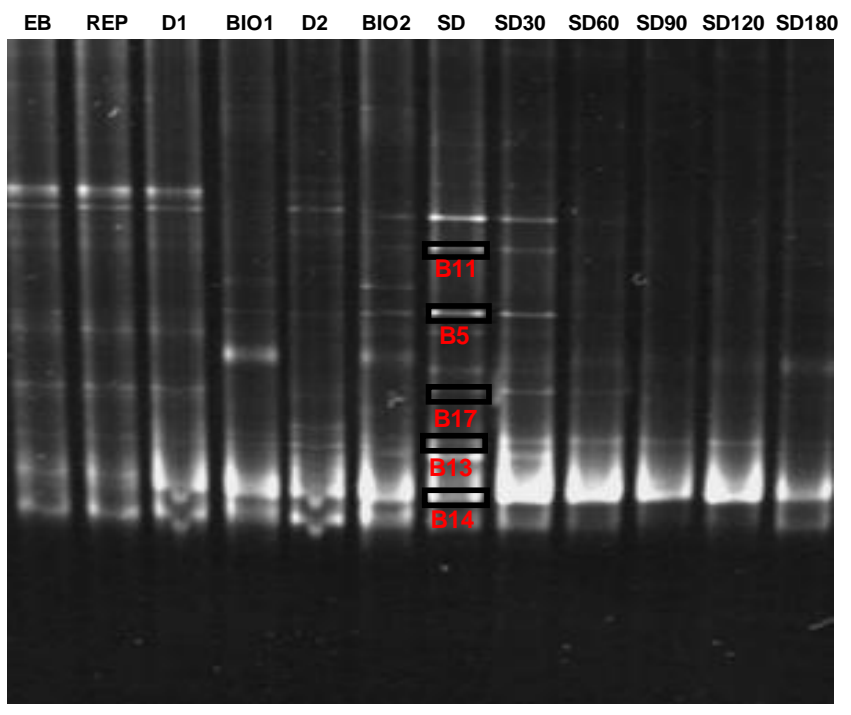

(B)

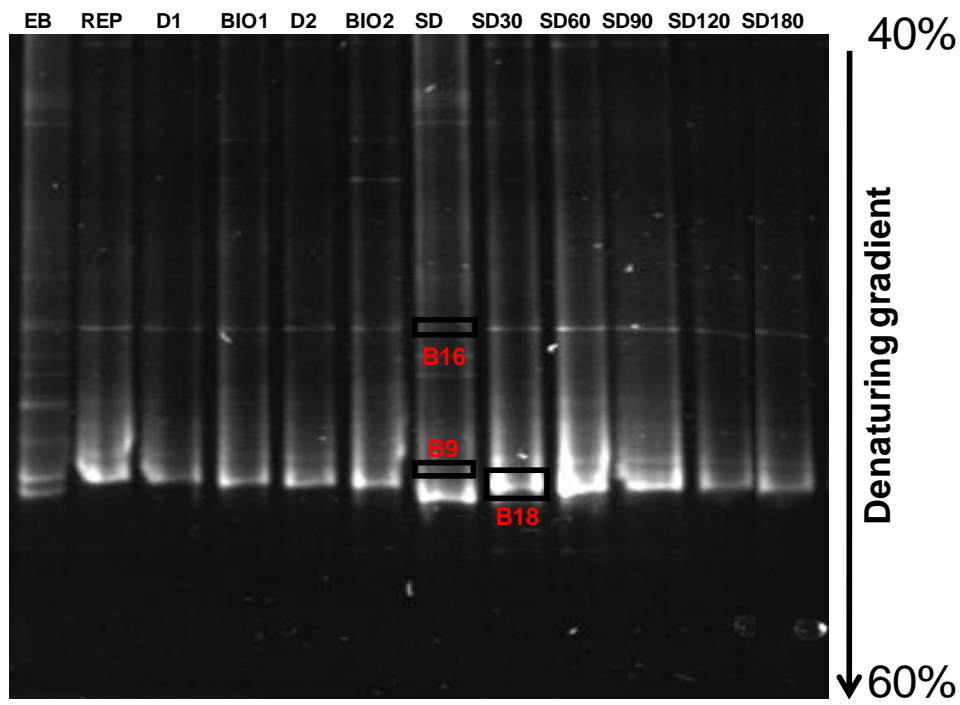

Figure 2. DGGE patterns of V3-V5 region of the 16S rDNA generated from the wastewater and biofilm samples collected in summer (A) and winter (B), from different sites in the RBC system. EB: entrance of the primary settlement tank, REP: outlet part of the primary settlement tank, D1: entrance of the first RBC tank, D2: outlet part of the second RBC tank, SD: outlet part of the secondary settling tank, BIO1 and BIO2: biofilm samples taken from the first and the second disc, respectively. SD30, SD60, SD90, SD120 and SD180: DGGE patterns of $16 \mathrm{~S}$ rDNA fragments amplified from secondary treated wastewater exposed to increasing UV253.7 Germicidal doses. (SD: output secondary clarifier ( $\left.T_{0}\right), \mathrm{s}$ : seconde) Bands marked were excised and sequenced. The gradient of the urea and formamide ranged from 40 to $60 \%$.

analysis. The results of homology search and the origin of the closet relative for the sequences obtained were showed in Table 2. The recovered fragment sequence of band B5 has a high similarity (99\%) to an uncultured Cyanobacterium isolated from cultivated soil (Jangid et al., 2011).

Band B9 have $99 \%$ similarity on the 16S rRNA gene level with Salmonella enterica (Hurrell et al., 2009). Band B11 is $100 \%$ similar with Fluviicola taffensis, a novel freshwater bacterium of the family Cryomorphaceae in the phylum Bacteroidetes, isolated from water of the River Taff, Cardiff, UK (O'Sulivan et al., 2005). Band B13 and B17 showed 99\% of homology with Chromobacterium sp. Strain DS1, a novel cholesterol oxidase producer (Doukyu et al., 2008). In addition these sequences have a high similarity $(99 \%)$ to Chromobacterium violaceum strain 968 (Wesener et al., 2011). The $16 \mathrm{~S}$ rDNA sequence of band $B 14$ has a high similarity (99\%) to Aquitalea sp. strain AOLR28 isolated from aerobic sludge granules (Aday et al., 2010). Finalely, two bands B16 and B18 were exclusively obtained from samples collected in winter.

Band B16 is $99 \%$ similar to Burkholderia sp. strain M27-VN8-1W, which was isolated from Vietnamese soils contaminated with 2,4-Dichlorophenoxyacetic acid (2,4D) and 2,4,5-Trichlorophenoxyacetic acid (2,4,5-T), (Huong et al., 2007). It is probably that the corresponding organism of band B16 is Burkholderia xenovorans which can grow at low temperature, near to $25^{\circ} \mathrm{C}$. (Rehmann and Dauguli, 2008; Hughes et al., 2011). The 16S rDNA sequence of band B18 has $100 \%$ of homology with Pantoea agglomerans which was isolated from activated sludge (Parsley et al., 2010).

\section{Denaturing gradient gel electrophoresis (DGGE) patterns analysis of ultraviolet (UV)-C radiation wastewater treatment}

The persistence of pathogens in secondary treated wastewater (the outlet part of the secondary settling tank) stresses the need for tertiary treatment as disinfection UV irradiation at $253.7 \mathrm{~nm}$. For this reason, we treated wastewater samples with varying doses of $\mathrm{UV}_{253.7}$. Comparison of DGGE patterns obtained in summer and winter seasons demonstrated reduction of number and intensity of bands by increasing of exposure UV-C dose (Figure 2).

In summer, the secondary treated DGGE pattern enclosed 8 visible bands including 5 bands (B5, B11, $B 13, B 14$ and B17), which were sequenced and identified. From UV dose equal to $720 \mathrm{~mW} . \mathrm{s.cm}^{-2}$ (60s irradiation), the band B5, B11 and B17 corresponding to Cyanobacterium, Fluviicola taffensis and Chromobacterium sp., respectively disappeared completely. For the remaining band B13, high UV-C dose $\left(\geq 2160 \mathrm{~mW} . \mathrm{s} . \mathrm{cm}^{-2}\right)$ reduced considerably bands intensity, suggesting the possible diminution and/or disap- 
Table 1. Shannon diversity index calculated from DGGE data. $\mathrm{S}$ : samples collected in summer, W: samples collected in winter.

\begin{tabular}{lcc}
\hline Sample & Band number & Shannon diversity index $(\boldsymbol{H})$ \\
\hline EBs & 10 & 2.303 \\
REPs & 10 & 2.079 \\
D1s & 10 & 2.079 \\
BIO1s & 6 & 1.792 \\
D2s & 8 & 2.079 \\
BIO2s & 9 & 2.197 \\
SDs & 9 & 2.197 \\
SD30s & 9 & 2.197 \\
SD60s & 5 & 1.609 \\
SD90s & 4 & 1.386 \\
SD120s & 4 & 1.386 \\
SD180s & 3 & 1.099 \\
Average & $\sim 7$ & 1.86 \\
EBw & 9 & 2.197 \\
REPw & 2 & 0.693 \\
D1w & 2 & 0.693 \\
BIO1w & 4 & 1.386 \\
D2w & 3 & 1.099 \\
BIO2w & 4 & 1.386 \\
SDw & 6 & 1.792 \\
SD30w & 3 & 1.099 \\
SD60w & 2 & 0.693 \\
SD90w & 2 & 0.693 \\
SD120w & 2 & 0.693 \\
SD180w & 2 & 0.693 \\
Average & $\sim 3$ & 1.09 \\
\hline & &
\end{tabular}

pearance of these populations in UV-C treated wastewater. However, we have noticed the persistence of the high intensity of the band B14 even after the use of UV dose equal to1440 mW.s.cm $\mathrm{cm}^{-2}$.

In winter, the secondary treated wastewater DGGE pattern enclosed three visible bands including bands B9, $\mathrm{B} 16$ and B18. Bands B9 and B18 related to Salmonella sp. and $P$. agglomerans, respectively were very weak. However, the use of UV dose equal to $360 \mathrm{~mW} . \mathrm{s}^{\mathrm{cm}}{ }^{-2}(\geq$ $30 \mathrm{~s}$ irradiation) increased the intensity of these two bands, and their diminution was from 1440 and 2160 mW.s. $\mathrm{cm}^{-2}$, respectively (Table 2 ). The band B16 persisted even after the use of UV dose equal to 2160 mW.s.cm ${ }^{-2}$.

At wavelength 253.7, UV irradiation can denature DNA (Zimmer and Slawson, 2002), or even cause structural changes inducing loss of vital cellular compounds, which leads to inhibition of replication, loss of reproducibility, and cell death (Liu et al., 1993; Nigro et al., 1998; Ben said et al., 2012). Indeed, obtained results showed varia- bility in bacterial tolerance to $\mathrm{UV}_{253.7}$ radiation. This variability is inter-specific and is dependent on the UV dose used. Accordingly, the effectiveness of UV disinfection depends on two factors: (i) the $U^{253.7}$ doses used and (ii) the irradiated bacterial species. Similar results reported by Hassen et al. (2000) showed that the susceptibility of bacteria to $\mathrm{UV}_{253.7}$ radiation was different from one specie to another and that UV is more effective on Gram-negative strains.

An increase in the intensity of bands after UV irradiation was observed for Salmonella sp., Aquitalea sp. and $P$. agglomerans. This result is probably due to the bacterial reactivation in the presence of visible light. Indeed, in response to moderate and/or non-lethal UV-C dose, bacteria react by mechanisms of DNA repair. Several studies showed that, to a certain extent, DNA damage can be tolerated by the cell until repair occurs (Lindauer and Darby, 1994; Arrieta et al., 2000; Zimmer and Slawson, 2002). The mechanism by which, microorganism recovers replication activity is called photoreactivation (Douki et al., 2003). Apart from photoreactivation numerous light-independent repair mechanisms exist that are regulated by the expression of the single-strand DNA binding protein RecA (Makarova et al., 2000). This result underscored the importance of bacterial mechanisms that could be used to overcome stress conditions generated by moderate UV exposure.

Finally, this study shows that the elimination of the majority of pathogenic bacteria in wastewater required an $\mathrm{UV}_{253.7}$ dose higher than $2160 \mathrm{~mW} . \mathrm{s.cm}^{-2}$ which is recommended for this type of treatment.

The culture-independent technique allows characterization of bacterial communities present in a sample without resorting to traditional microbiological methods (Jany and Barbier, 2008) and has a major advantage as evidenced by its ability to detect non-cultivable bacterial species or difficult to identify by conventional culture methods (Evans et al., 2004). Especially, when the irradiated samples included different bacterial viability form among the same irradiated bacterial specie, as viable but non cultivable (ABNC) bacteria not yet reactivated, active but non cultivable (ABNC) bacteria and VBNC-UV inactivated bacteria (Ben Said et al., 2012).

Consequently, denaturing gradient gel electrophoresis method (DGGE) provided precise information for the effect of increasing $U_{253.7}$ germicidal dose on the bacterial community in secondary treated wastewater effluent, and could be useful for studying the effect of different wastewater treatment processes on the bacterial community.

\section{Conclusion}

Rotating biological contactor has been frequently used for the biological wastewater treatment in order to remove 
Table 2. Effect of increasing UV253.7 Germicidal doses on bacterial species of secondary treated wastewater and the partial sequences analysis of (16S rDNA genes) major bands recovered from DGGE pattern.

\begin{tabular}{|c|c|c|c|c|c|c|c|c|c|}
\hline \multirow{3}{*}{ Bande } & \multirow{3}{*}{$\begin{array}{l}\text { rDNA accession } \\
\text { number }\end{array}$} & \multirow{3}{*}{ Closed species } & \multirow[t]{3}{*}{ Similarity (\%) } & \multicolumn{6}{|c|}{ Exposure time (s) and UV253,7 doses (mW. S. $\left.\mathrm{cm}^{-2}\right)$} \\
\hline & & & & $0(s)$ & 30 & 60 & 90 & 120 & 180 \\
\hline & & & & 0 (mW. S. $\left.\mathrm{cm}^{-2}\right)$ & 360 & 720 & 1080 & 1440 & 2160 \\
\hline B5 & JQ003547 & Uncultred Cyanobacterium & 99 & + & + & - & - & - & - \\
\hline B9 & JN193512 & Salmonella enterica & 100 & $+/-$ & $+/-$ & + & $+/-$ & - & - \\
\hline B11 & JN193514 & Fluviicola taffensis & 100 & + & + & - & - & - & - \\
\hline B13 & JN193516 & Chromobacterium sp. & 99 & + & + & + & + & + & + \\
\hline B14 & JN193517 & Aquitalea sp. & 99 & + & ++ & ++ & ++ & ++ & + \\
\hline B16 & JN193519 & Burkholderia sp. & 99 & + & + & + & + & $+/-$ & $+/-$ \\
\hline B17 & JN193520 & Chromobacterium sp. & 99 & $+/-$ & $+/-$ & - & - & - & - \\
\hline B18 & JQ003548 & Pantoea agglomerans sp. & 100 & $+/-$ & ++ & ++ & ++ & + & $+/-$ \\
\hline
\end{tabular}

Intensity of band are (-): absence, (+/-): low, (+): high, (++): very high.

pollutants and improve the water quality. The presence of bacteria species in the secondary treated wastewater indicate the necessity of a tertiary treatment process to reduce the number of living organisms in the water to be discharged. Obtained results show that DGGE patterns of samples collected in summer were more complex than those collected in winter. In addition, variability in bacterial tolerance to $U V_{253.7}$ radiation was revealed. This variability is inter-specific and is depen-dent on the UV-C dose used and the bacterial species irradiated. Consequently, the DGGE technique coupled with sequencing provides precise information on UV-C wastewater treatment process, and could be of great importance to wastewater treatment studies.

\section{REFERENCES}

Aday SS, Lee DJ, Lai JY (2010). Potential cause of aerobic granular sludge breakdown at high organic loading rates. Appl Microbiol Biotechnol. 85:1601-1610.

Arrieta JMA, Weinbauer MG, Herndl GJ (2000). Interspecific variability in sensitivity to UV radiation and subsequent recovery in selected isolates of marine bacteria. Appl. Environ. Microbiol. 66:1468-1473.

Ben Said M, Khefacha S, Maalej L, Hassen A (2011). Effect of ultraviolet, electromagnetic radiation subtype C (UV-C) dose on biofilm formation by Pseudomonas aeruginosa, Afr. J. Microbiol. Res. 5:4353-4358.

Ben said M, Otaki M, kazama S, Hassen A (2012). Development of a DNA-dosimeter system as biomarker to monitor the effects of pulsed ultraviolet radiation, Afr. J. Microbiol. Res. 6:5153-5161.

Ding L, Zhou Q, Wang L, Zhang Q (2011). Dynamics of bacterial community structure in a fullscale wastewater treatment plant with anoxic-oxic configuration using 16S rDNA PCR-DGGE fingerprints. Afr J Biotechnol. 10:589-600.

Douki T, Laporte G, Cadet J (2003). Inter-stand photoproducts are produced in high yield within A-DNA exposed to UVC radiation. Nucleic Acids Res. 31 (12):3134-3142.

Doukyu N, Shibata K, Ogino H, Sagermann M (2008). Purification and characterization of Chromobacterium sp. DS-1 cholesterol oxidase with thermal, organic solvent, and detergent tolerance. Appl. Microbiol. Biotechnol. 80:59-70.

Eccleston B (1998). UV intensity levels affected by water quality. Water Technol. 21:61-68.
Evans FF, Seldin L, Sebastian GV, Kjelleberg S, Holmstro C, Rosado AS (2004). Influence of petroleum contamination and biostimulation treatment on the diversity of Pseudomonas spp. in soil microcosms as evaluated by $16 \mathrm{~S}$ rRNA based-PCR and DGGE. Lett. Appl. Microbiol. 38:93-98.

Gilbride KA, Frigon D, Cesnik A, Gawat J, Fulthorpe RR (2006). Effect of chemical and physical parameters on a pulp mill biotreatment bacterial community. Water Res. 40:775-87.

Guillaume G, Verbrugge D, Chasseur-Libotte M, Moens W, Collard J (2000). PCR typing of tetracycline resistance determinants (Tet A-E) in Salmonella enterica serotype Hadar and in the microbial community of activated sludges from hospital and urban wastewater treatment facilities in Belgium. FEMS Microbiol. Ecol. 32:77-85.

Hassen A, Mahrouk M, Ouzari H, Cherif A, Boudabous A, Damelincourt JJ (2000).UV disinfection of treated wastewater in a large scale pilot plant and inactivation of selected bacteria in a laboratory UV device. Bioresour. Technol. 74:141-150.

Howard I, Espigares E, Lardelli p, Martin JL, Espigares M (2004). Evaluation of microbiological and physicochemical indicators for wastewater treatment. Environ. Toxicol. 19:241-249.

Hughes D, Clarck B, Murphy CD (2011). Bioremediation of polyfluorinated biphenyl in bacteria. Biodegradation 22:41-749.

Huong N, Itoh K, Suyama K (2007). Diversity of 2,4dichlorophenoxyacetic acid (2,4-D) and 2,4,5-trichlorophenoxyacetic acid (2 4,5-T)-degrading bacteria in Vietnams soils. Microbes Environ. 22:243-256.

Hurrell E, Kucerova E, Loughlin M, Caubilla-Barron J, Forsythe SJ (2009). Biofilm formation on enteral feeding tubes by Cronobacter sakazakii, Salmonella serovars and other Enterobacteriaceae. Int. J. Food Microbiol. 136:227-231.

Jangid K, Williams MA, Franzluebbers AJ, Schmidt TM, Coleman DC, Whitman WB (2011). Land-use history has a stronger impact on soil microbial community composition than aboveground vegetation and soil properties. Soil Biol. Biochem. 43:2184-2193.

Jany JL, Barbier G (2008). Culture-independent methods for identifying microbial communities in cheese. Food Microbiol. 25:839-848.

Lindauer KG, Darby J (1994). Ultraviolet disinfection of waste water: effect of dose on subsequent photoreactivation. Water Res. 28:805817.

Liu WK, Tebbs SE, Byrne PO, Elliott TSJ (1993). The effects of electric current on bacteria colonising intravenous catheters. J. Infect. 27:261-269

Makarova O, Kamberov E, Margolis B (2000). Generation of deletion and point mutations with one primer in a single cloning step. Biotech. 29:970-972.

Marzorati M, Alma A, Sacchi L, Pajoro M, Palermo S, Brusetti L, Raddadi N, Balloi A, Tedeschi R, Clementi E, Ccorona S, Quaglino F, Bianco PA, Beninati T, Bandi C, Daffonchio D (2006). 
A novel Bacteroidetes symbiont is localized in Scaphoideus titaneus, the insect vector of Flavescence dorée in Vitis vinifera. Appl. Environ. Microbiol. 72:1467-1475.

Moura A, Tacao M, Heneriques I, Dias J, Ferreira P, Correia A (2009). Characterization of bacterial diversity in two aerated lagoons of a wastewater treatment plant using PCR-DGGE analysis. Microbiol Res. 164:560-569.

Muyzer G, Dewaal EC, Uitterlinden AG (1993). Profiling of complex microbial populations by denaturing gradient gel electrophoresis of polymerase chain reaction-amplified genes coding for 16S rRNA. Appl Environ Microbiol. 59:695-700.

Nigro F, Ippolito A, Lima G (1998). Use of UV-C light to reduce Botrytis storage rot of table grapes. Postharvest Biol. Tech. 13:171-181.

O'Sulivan LA, Rinna J, Humphreys G, Weightman AJ, Fry JC (2005). Fluviicola taffensis gen. nov., sp. nov., a novel freshwater bacterium of the family Cryomorphaceae in the phylum 'Bacteroidetes'. Int. J. Syst. Evol. Microbiol. 55:2189-2194.

Parsley LC, Consuegra EJ, Thomas SJ, Bhavsar J, Land AM, Bhuiyan NN, Mazher MA, Waters RJ, Wommack KE, Happer W, Liles MR (2010). Census of the viral metagenome within an activated sludge microbial assemblage. Appl. Environ. Microbiol. 76:2673-2677.

Rehmann L, Dauguli AJ (2008). Biodegradation of PCBs in two-phase partitioning bioreactors following solid extraction from soil. Biotechnol Bioeng. 99:1273-1280.
Sass AM, Sass H, Coolen MJ, Cypionka H, Overmann J (2001). Microbial communities in the chemocline of a hypersaline deep-sea basin (Urania basin, Mediterranean Sea). Appl Environ Microbiol. 67:5392-5402.

Steele JA, Ozis F, Fuhrman JA, Devinny JS (2005). Structure of microbial communities in ethanol biofilters. Chem Eng J. 113:135143.

Wesener SR, Potharla VY, Cheng YQ (2011). Reconstitution of the FK228 biosynthetic pathway reveals cross talk between modular polyketide synthases and fatty acid synthase. Appl. Environ. Microbiol. 77:1501-1507.

Zimmer JL, Slawson RM (2002). Potential repair of Escherichia coli DNA following exposure to UV radiation from both medium- and lowpressure UV sources used in drinking water treatment. Appl. Environ. Microb. 68:3293-3299. 